\title{
FINITE SEMILATTICES WHOSE MONOIDS OF ENDOMORPHISMS ARE REGULAR
}

\author{
M. E. ADAMS AND MATTHEW GOULD \\ Dedicated to the memory of $G$. H. Wenzel
}

\begin{abstract}
A classification is obtained for the finite semilattices $S$ such that the monoid of endomorphisms of $S$ is regular in the semigroup-theoretic sense.
\end{abstract}

\section{INTRODUCTION}

An element $f$ of a semigroup $M$ is said to be regular if there exists $g \in M$ such that $f=f g f$; if each element of $M$ is regular, $M$ itself is said to be regular. Regular semigroups have long been a mainstay of semigroup theory (see, for example, [6]), and in particular there are a number of papers investigating regularity for the monoid (semigroup with identity) $\operatorname{End}(A)$ of endomorphisms of an algebraic or relational system $A$. Such an investigation would seem to be especially meaningful and feasible for classes $\mathbf{K}$ of algebraic or relational systems that are uniquely recoverable from their endomorphism monoids in the sense that $A \cong B$ whenever $A, B \in \mathbf{K}$ and $\operatorname{End}(A) \cong \operatorname{End}(B)$. For example, Schein [7] established unique recoverability for several classes of algebras, including the class of all semilattices. In [4], Gluskin showed that posets are almost uniquely recoverable, in the sense that the endomorphism monoid of a poset determines the poset up to isomorphism or dual-isomorphism.

Posets $P$ for which $\operatorname{End}(P)$ is regular were characterized by Aizenshtat [2]. (The present authors [1] also characterized such posets, unwittingly duplicating Aizenshtat's characterization in the case of posets that are not chains.) Unars with regular endomorphism monoids were characterized by Skornjakov [8] and Chvalina [3].

The goal of the present work is to determine all finite semilattices $S$ such that $\operatorname{End}(S)$ is regular.

The major results utilize the concepts of vertical and horizontal sum of finite lattices $L_{i}, i<n$, where $n>1$. The vertical sum (see $\S 4$ ) is formed by identifying the unit of $L_{i}$ (for $i<n-1$ ) with the zero of $L_{i+1}$ in the ordinal sum; the horizontal sum (see $\S 5$ ) is formed by adjoining a zero and a unit to the disjoint union of the lattices.

Let $\mathbf{B}$ denote the class of all vertical sums of finite lattices of the form $B \cup\{1\}$ where $B$ is either empty or a finite binary tree (see $\S 2)$. Let $\mathbf{B}^{d}$ be the class

Received by the editors August 3,1989 and, in revised form, May 10, 1990.

1980 Mathematics Subject Classification (1985 Revision). Primary 06A12, 20M20; Secondary 08A35, $20 \mathrm{M} 17$. 
of all lattices $L$ such that the dual of $L$ lies in $\mathbf{B}$. Finally, let $\mathbf{R}$ denote the smallest class of lattices that contains the one- and two-element chains and is closed under the operations of vertical and horizontal sum.

Our major results are all given in $§ 7$. The principal result (Theorem 7.1) is the following. Let $S$ be a finite semilattice. Then $\operatorname{End}(S ; \wedge)$ is regular if and only if either $S$ is a binary tree, $S$ is a tree with only one $\wedge$-reducible element, or $S$ is bounded and, as a lattice, $S \in \mathbf{B} \cup \mathbf{B}^{d} \cup \mathbf{R}$.

Prior to $\S 7$, the paper consists entirely of those various parts that combine to give Theorem 7.1. It is arranged as follows. In $\S 2$ it is shown that, for a finite semilattice $S$, if $\operatorname{End}(S ; \wedge)$ is regular, then $S$ must be either a tree or (the $\wedge$ semilattice of) a lattice (Proposition 2.1). Further, the regular endomorphisms of semilattices are characterized (Proposition 2.2). This characterization is used repeatedly throughout the remainder of the paper. In $\S 3$ it is shown that, for a finite semilattice $S$, if $S$ is a tree, then $\operatorname{End}(S)$ is regular if and only if $S$ is binary or has only one $\wedge$-reducible element (Proposition 3.6). In $\S 4$ it is shown that, if $S \in \mathbf{B} \cup \mathbf{B}^{d}$, then $\operatorname{End}(S ; \wedge)$ is regular (Propositions 4.3 and 4.5). In $\S 5$ it is shown that, if $S \in \mathbf{R}$, then $\operatorname{End}(S ; \wedge)$ is regular (Proposition 5.2). Finally, in $\S 6$ it is shown (Propositions 6.2 and 6.8) that, if $S$ is a finite lattice and $\operatorname{End}(S ; \wedge)$ is regular, then $S \in \mathbf{B} \cup \mathbf{B}^{d} \cup \mathbf{R}$.

A surprising and immediate consequence of Theorem 7.1 is that, for a finite lattice $S, \operatorname{End}(S ; \wedge)$ is regular if and only if $\operatorname{End}(S ; \vee)$ is regular (Corollary 7.2). Moreover, to derive Theorem 7.1, a set of 40 lattices is exhibited such that $\mathbf{B} \cup \mathbf{B}^{d} \cup \mathbf{R}$ consists precisely of those finite lattices having no sublattice isomorphic to a member of the set (see $\S 6$ ). This, in turn, provides an alternate characterization of those finite semilattices whose endomorphism monoids are regular (Theorem 7.3).

Recently, Janowitz [5] has considered the regularity of residuated maps of complete lattices. It is interesting that his results and ours bear a superficial resemblance in their statement.

\section{Preliminaries}

A semilattice $(S ; \wedge)$ is a commutative semigroup in which each element is idempotent. A partial ordering is defined on $S$ by $a \leq b$ if and only if $a \wedge b=a$; with respect to this order, each pair of elements of $S$ has a greatest lower bound, or meet, which coincides with the operation $\wedge$. If each pair of elements of $S$ also has a least upper bound, or join (denoted $\vee$ ), then $S$ is said to be a lattice. (More properly: $(S ; \vee, \wedge)$ is a lattice.)

Elements $a, b$ of a semilattice $S$ are comparable if $a \wedge b \in\{a, b\}$; if $a$ and $b$ are not comparable, we write $a \| b$. A $\wedge$-reducible element is one that can be expressed as $a \wedge b$ where $a \| b$. (In the event that $S$ is a lattice, then a $\vee$-reducible element is one that can be written as $a \vee b$ for some $a \| b$.) A subset $C$ of $S$ for which all $a, b \in C$ are comparable is called a chain. An antichain is a subset $A$ of $S$ such that $a \| b$ for all distinct $a, b \in A$.

For an element $a$ of a semilattice $S$, the principal ideal generated by $a$ is the set $(a]=\{x \in S: x \leq a\}$, and the principal filter generated by $a$ is the set $[a)=\{x \in S: x \geq a\}$. If $(a]$ is a chain for all $a \in S$, then $S$ is said to be a tree. A tree is said to be binary if for each $\wedge$-reducible $a$ there are precisely two elements that cover $a$. (An element $a$ is said to cover an element $b$, denoted 
$a \succ b$, if $a>b$ and there is no $c$ satisfying $a>c>b$.) Note that every chain is a binary tree.

The smallest element of a finite semilattice $S$ is denoted 0 . If $S$ has a largest element, it is denoted 1 and $S$ is said to be bounded. A finite semilattice is a lattice if and only if it is bounded.

Proposition 2.1. Let $S$ be a finite semilattice such that $\operatorname{End}(S)$ is regular. Then $S$ is either a tree or a lattice.

Proof. To prove the contrapositive, suppose $S$ is neither a tree nor a lattice. Then there exist $a, b, c \in S$ with $a \leq c, b \leq c$, and $a \| b$, and by finiteness there exist $u, v \in S$ such that $\{u, v\}$ has no upper bound. Because $[u) \cap[v)=$ $\varnothing$, a map $f \in \operatorname{End}(S)$ can be defined by

$$
f(x)= \begin{cases}a & \text { if } x \in[u), \\ b & \text { if } x \in[v), \\ a \wedge b & \text { otherwise. }\end{cases}
$$

If there existed $g \in \operatorname{End}(S)$ satisfying $f=f g f$, then $g(c)$ would be an upper bound for $\{u, v\}$. Thus $\operatorname{End}(S)$ is not regular.

The following proposition, although stated for semilattices, holds for any algebraic system. It characterizes regularity of an endomorphism in terms of the concepts of retraction and faithful pre-image. A retraction is an idempotent endomorphism, that is, an endomorphism that fixes each element of its image. The image of a retraction is called a retract of the domain. Given a semilattice $T$, an endomorphism $f$ of $T$, and a subsemilattice $S$ of $\operatorname{Im}(f)$ (the image of $f$ ), a faithful pre-image of $S$ by $f$ is a subsemilattice $S^{\prime}$ of $T$ such that the restriction of $f$ to $S^{\prime}$ (denoted $f \mid S^{\prime}$ ) is an isomorphism of $S^{\prime}$ onto $S$ : equivalently, $S^{\prime}$ is a subsemilattice of $f^{-1}(S)$ and $\left|S^{\prime} \cap f^{-1}\{x\}\right|=1$ for all $x \in S$.

Proposition 2.2. An endomorphism $f$ of a semilattice $S$ is regular if and only if the following hold.

(i) There is a retraction of $S$ onto $\operatorname{Im}(f)$.

(ii) There is a faithful pre-image of $\operatorname{Im}(f)$ by $f$.

Proof. If $f$ is regular, then $\operatorname{Im}(f)=\operatorname{Im}(f g)$ and $(f g)^{2}=f g$, where $g \in$ $\operatorname{End}(S)$ satisfies $f g f=f$. Hence $f g$ is a retraction establishing (i). To verify (ii), set $R=\operatorname{Im}(g f)$. Then $f g f=f$ implies $\operatorname{Im}(f)=\operatorname{Im}(f \uparrow R)$ and $f \uparrow R$ is one-to-one.

Conversely, assuming (i) and (ii) hold, choose a retraction $\rho$ and a faithful pre-image $S^{\prime}$, and set $g=\left(f \uparrow S^{\prime}\right)^{-1} \rho$. Then $g \in \operatorname{End}(S)$ and $f g f=f$.

The following lemma, which is well known and easily proved, will be used several times in the sequel.

Lemma 2.3. If $L_{1}$ is a lattice containing a finite sublattice $L_{0}$, a retraction $\rho$ of $\left(L_{1} ; \wedge\right)$ onto $\left(L_{0} ; \wedge\right)$ is given by $\rho(x)=\bigvee\left((x] \cap L_{0}\right)$ when $(x] \cap L_{0} \neq \varnothing$ and $\rho(x)=\bigwedge L_{0}$ otherwise.

\section{TREES}

The goal of this section is to determine the finite trees $S$ for which $\operatorname{End}(S)$ is regular. 
Given a semilattice $S$ and a class $\mathbf{K}$ of semilattices, $S$ is said to be faithfully pre-imaged over $\mathbf{K}$ if there exists a faithful pre-image of $S$ by $f$ whenever $f \in \operatorname{End}(T)$ for some $T \in \mathbf{K}$ and $S$ is a subsemilattice of $\operatorname{Im}(f)$.

Lemma 3.1. Every finite chain is faithfully pre-imaged over the class of all semilattices.

Proof. Let $T$ be a semilattice, let $f \in \operatorname{End}(T)$, and let $S$ be a finite chain in $\operatorname{Im}(f)$. For each $a \in S$ choose $a^{*} \in f^{-1}\{a\}$ and define

$$
a^{\prime}=\bigwedge\left(b^{*}: b \in S \cap[a)\right) \text {. }
$$

Then $\left\{a^{\prime}: a \in S\right\}$ is a faithful pre-image of $S$ by $f$.

A semilattice $S$ is said to satisfy the strong meet property if $a_{0} \wedge a_{1}=b_{0} \wedge b_{1}$ whenever $a_{0}, a_{1}, b_{0}, b_{1}$ are elements of $S$ such that $a_{0} \| a_{1}$ and $b_{i} \in\left[a_{i}\right) \backslash\left[a_{1-i}\right)$ for $i=0,1$. Note that if $S$ is a tree, it is equivalent to assert that $a_{0} \wedge a_{1}=$ $b_{0} \wedge b_{1}$ whenever $a_{0} \| a_{1}$ and $b_{i} \in\left[a_{i}\right), i=0,1$.

Lemma 3.2. Every finite binary tree is faithfully pre-imaged over the class of all semilattices that satisfy the strong meet property.

Proof. Let $S$ be a finite binary tree. As the statement is trivial for $|S|=1$, we proceed by induction, assuming $|S|>1$ and the statement holds for all binary trees of smaller cardinality.

Let $T$ be a semilattice satisfying the strong meet property, and let $f \in$ $\operatorname{End}(T)$ be such that $S$ is a subsemilattice of $\operatorname{Im}(f)$. By Lemma 3.1, we may assume that $S$ has at least one $\wedge$-reducible element. Let $a$ be the smallest $\wedge$-reducible element of $S$, and let $a_{0}$ and $a_{1}$ be the covers of $a$ in $S$. Then $S=A_{0} \cup A_{1} \cup A_{2} \cup\{a\}$, where $A_{i}=\left[a_{i}\right) \cap S$ for $i=0,1$, and $A_{2}=((a] \backslash\{a\}) \cap S$.

By the induction hypothesis, for each $i<3$ (or $i<2$, if $A_{2}=\varnothing$ ), let $B_{i}$ be a faithful pre-image of $A_{i}$ by $f$. For $i=0,1$ let $b_{i} \in B_{i}$ be such that $f\left(b_{i}\right)=a_{i}$. If $A_{2}=\varnothing$, set $B_{2}=\varnothing$.

Set $B=B_{0} \cup B_{1} \cup\left\{b_{0} \wedge b_{1}\right\} \cup\left\{x \wedge b_{0} \wedge b_{1}: x \in B_{2}\right\}$. Because $f\left(b_{0} \wedge b_{1}\right)=a$ and $f\left(x \wedge b_{0} \wedge b_{1}\right)=f(x)$ for all $x \in B_{2}$, to show that $B$ is a faithful pre-image of $S$ it suffices to verify that $c_{0} \wedge c_{1} \in B$ whenever $c_{i} \in B_{i}, i=0,1$. Note that $b_{0} \| b_{1}$ because $a_{0} \| a_{1}$. Clearly $c_{i} \in\left[b_{i}\right)$ for $i=0,1$. Moreover, $c_{i} \notin\left[b_{1-i}\right)$ because $f\left(c_{i}\right) \notin\left[a_{1-i}\right)$. Thus the strong meet property gives $c_{0} \wedge c_{1}=b_{0} \wedge b_{1} \in B$.

Lemma 3.3. Every tree satisfies the strong meet property.

Proof. Let $a_{0}, a_{1}, b_{0}, b_{1}$ be elements of a tree $S$ satisfying $a_{0} \| a_{1}$ and $b_{i} \in$ $\left[a_{i}\right), i=0,1$. Clearly $a_{0} \wedge a_{1} \leq b_{0} \wedge b_{1}$. For fixed $i=0,1$, the elements $a_{i}$ and $b_{0} \wedge b_{1}$ are comparable because they are members of the chain $\left(b_{i}\right]$. If $a_{i} \leq b_{0} \wedge b_{1}$, then $\left\{a_{i}, a_{1-i}\right\} \subseteq\left(b_{1-i}\right]$, which is impossible because $a_{i} \| a_{1-i}$. Hence $b_{0} \wedge b_{1} \leq a_{i}$. As this holds for both values of $i$, it follows that $b_{0} \wedge b_{1} \leq$ $a_{0} \wedge a_{1}$.

Lemma 3.4. Let $S$ be a semilattice having exactly one $\wedge$-reducible element. If $f \in \operatorname{End}(S)$, there is a faithful pre-image of $\operatorname{Im}(f)$ by $f$.

Proof. Let $a$ be the unique $\wedge$-reducible of $S$.

Suppose $f(a)=a$ and choose $x^{\prime} \in f^{-1}\{x\}$ for each $x \in \operatorname{Im}(f)$, such that $a^{\prime}=a$. Then $\left\{x^{\prime}: x \in \operatorname{Im}(f)\right\}$ is a faithful pre-image of $\operatorname{Im}(f)$ by $f$.

Suppose now that $f(a) \neq a$. It suffices by Lemma 3.1 to show that $\operatorname{Im}(f)$ is a chain. If there exist $x, y \in S$ with $f(x) \| f(y)$, then $f(x) \wedge f(y)=a$. 
But $f(x) \| f(y)$ implies $x \| y$, whence $f(x) \wedge f(y)=f(x \wedge y)=f(a) \neq a$, a contradiction.

Lemma 3.5. If $S$ is a tree and $T$ a finite subsemilattice of $S$, there is a retraction of $S$ onto $T$.

Proof. Let $S^{1}$ be the semilattice obtained by adjoining a unit to $S$. Then $S^{1}$ is a lattice containing $T^{1}=T \cup\{1\}$ as a sublattice. The retraction $\rho$ of $\left(S^{1} ; \wedge\right)$ onto $\left(T^{1} ; \wedge\right)$ given by Lemma 2.3 has the property that $\rho(x) \neq 1$ for all $x \in S$. Hence $\rho \mid S$ is a retraction of $S$ onto $T$.

We can now characterize those finite trees whose endomorphism monoids are regular.

Proposition 3.6. For a finite tree $S, \operatorname{End}(S)$ is regular if and only if $S$ is binary or has exactly one $\wedge$-reducible element.

Proof. Let $f \in \operatorname{End}(S)$, where $S$ is a finite tree that is binary or has exactly one $\wedge$-reducible. If $S$ is binary, then $\operatorname{Im}(f)$ is also a binary tree. Thus in either case there is a faithful pre-image of $\operatorname{Im}(f)$ by $f$, obtained via Lemmas 3.2 and 3.3, or via Lemma 3.4. A retraction of $S$ onto $\operatorname{Im}(f)$ is given by Lemma 3.5, whereupon $\operatorname{End}(S)$ is regular by Proposition 2.2.

Suppose $\operatorname{End}(S)$ is regular, where $S$ is not binary and has more than one $\wedge$-reducible. Let $a$ and $b$ be distinct $\wedge$-reducibles such that $a$ has at least three covers.

If $b>a$, choose distinct covers $b_{0}, b_{1}$ of $b$ and $a_{0}, a_{1}, a_{2}$ of $a$, such that $\left\{a_{1}, a_{2}\right\} \cap(b]=\varnothing$. Define $f \in \operatorname{End}(S)$ by $f\left(\left[b_{0}\right)\right)=\left\{a_{0}\right\}, f\left(\left[b_{1}\right)\right)=\left\{a_{1}\right\}$, $f\left(\left[a_{2}\right)\right)=\left\{a_{2}\right\}$, and $f(x)=a$ otherwise. If $g \in \operatorname{End}(S)$ satisfies $f=f g f$, then $g\left(a_{2}\right) \geq a_{2}$ and $g\left(a_{2}\right) \geq g(a)=g\left(a_{0}\right) \wedge g\left(a_{1}\right)$. Since $g\left(a_{i}\right) \geq b_{i}$ for $i=0,1$, it follows that $g\left(a_{2}\right) \geq b$, whence $a_{2}$ is comparable with $b$, a contradiction.

Hence $b \nsupseteq a$. Set $c=b \wedge a$. Then $c$ is $\wedge$-reducible and $c<a$. Choose distinct covers $c_{0}, c_{1}$ of $c$ and distinct covers $a_{0}, a_{1}, a_{2}$ of $a$ such that $a \nsupseteq$ $c_{1}$. Define $f \in \operatorname{End}(S)$ by $f\left(\left[a_{1}\right)\right)=\left\{a_{1}\right\}, f\left(\left[a_{2}\right)\right)=\left\{a_{2}\right\}, f\left(\left[c_{1}\right)\right)=\left\{a_{0}\right\}$, and $f(x)=a$ otherwise. If $g \in \operatorname{End}(S)$ satisfies $f=f g f$, then $g\left(a_{0}\right) \geq c_{1}$ and $g\left(a_{0}\right) \geq g(a)=g\left(a_{1}\right) \wedge g\left(a_{2}\right)$. Since $g\left(a_{i}\right) \geq a_{i}$ for $i=1,2$, it follows that $g\left(a_{0}\right) \geq a$, whence $c_{1} \nVdash a$, a contradiction.

\section{The Classes B AND $\mathbf{B}^{d}$}

In view of Proposition 2.1 and Theorem 3.6, the remaining task is to characterize the finite lattices $L$ for which $\operatorname{End}(L ; \wedge)$ is regular. These lattices fall naturally into three classes, two of which are defined in terms of capped binary trees and the operation of vertical sum.

A capped binary tree is the lattice obtained by adjoining a unit to a binary tree; for convenience the one-element chain (denoted $\mathbf{1}$ ) is also regarded as a capped binary tree. The vertical sum of bounded lattices $L_{0}$ and $L_{1}$ is defined (only up to isomorphism) by first replacing each $L_{i}$ by an isomorphic copy $L_{i}^{\prime}$ such that the unit of $L_{0}^{\prime}$ is the zero of $L_{1}^{\prime}$ and is the only element of $L_{0}^{\prime} \cap L_{1}^{\prime}$. A partial order is then defined on $L_{0}^{\prime} \cup L_{1}^{\prime}$ by retaining the ordering within each lattice and stipulating that $x \leq y$ whenever $x \in L_{0}^{\prime}$ and $y \in L_{1}^{\prime}$. The resulting lattice is denoted $L_{0}+{ }_{V} L_{1}$. (In practice, the distinction between $L_{i}$ and $L_{i}^{\prime}$ 
will be suppressed and $L_{i}$ will be regarded as a sublattice of $L_{0}+V L_{1}$.) Given bounded lattices $L_{i}, i<n$, where $n>1$, the vertical sum $\sum_{V}\left(L_{i}: i<n\right)$ is defined to be $\left(\cdots\left(\left(L_{0}+{ }_{V} L_{1}\right)+{ }_{V} L_{2}\right)+{ }_{V} \cdots\right)+{ }_{V} L_{n-1}$.

Let $\mathbf{B}$ denote the class of all vertical sums of finite capped binary trees, and let $\mathbf{B}^{d}$ denote the class of all lattices $L$ such that the dual of $L$ lies in $\mathbf{B}$. Because $L=L+{ }_{V} \mathbf{1}$ for every bounded lattice $L$, it is clear that every finite capped binary tree lies in $\mathbf{B}$. To establish the regularity of $\operatorname{End}(L ; \Lambda)$ for $L \in \mathbf{B} \cup \mathbf{B}^{d}$, we shall list some properties that are preserved by the operation of vertical sum.

Let $L$ be a finite lattice. A subsemilattice of $(L ; \wedge)$ is said to be a $\wedge$ subsemilattice of $L$. Likewise, by a $\wedge$-retract of $L$ is meant a retract of $(L ; \wedge)$. A bounded $\wedge$-subsemilattice $T$ of $L$ is said to be smooth if $T$ does not contain elements $a, b, c$ satisfying $c \| a \vee b$ and $c<a \vee_{T} b$, where $\vee_{T}$ denotes join with respect to $T$.

Lemma 4.1. Let $L_{i}, i<n$, where $n>1$, be finite lattices, and let $L=$ $\Sigma_{V}\left(L_{i}: i<n\right)$.

(i) If each $L_{i}$ satisfies the strong meet property, then so does $L$.

(ii) If each $\left(L_{i} ; \wedge\right)$ is faithfully pre-imaged with respect to a class $\mathbf{K}$ of semilattices, then so is $(L ; \wedge)$.

(iii) If, for all $i<n$, every smooth $\wedge$-subsemilattice of $L_{i}$ is a retract of $L_{i}$, then every smooth $\wedge$-subsemilattice of $L$ is a retract of $L$.

Proof. (i) Let $a_{j}, b_{j}, j=0,1$, be elements of $L$ such that $a_{0} \| a_{1}$ and $b_{j} \in$ $\left[a_{j}\right) \backslash\left[a_{1-j}\right)$. Since $b_{0} \| b_{1}$, there exist $k, l \in\{0, \ldots, n-1\}$ with $\left\{a_{0}, a_{1}\right\} \subseteq L_{k}$ and $\left\{b_{0}, b_{1}\right\} \subseteq L_{l}$. The condition $b_{j} \in\left[a_{j}\right) \backslash\left[a_{1-j}\right)$ forces $k=l$, whereupon the strong meet property in $L_{k}$ gives $a_{0} \wedge a_{1}=b_{0} \wedge b_{1}$ as desired.

(ii) It suffices to consider the case $n=2$, as the general proof would then be a straightforward induction.

Let $T \in \mathbf{K}$ and $f \in \operatorname{End}(T)$ such that $L \subseteq \operatorname{Im}(f)$. For each $i$ let $L_{i}^{\prime}$ be a faithful pre-image of $L_{i}$ by $f$, and let $p_{i} \in L_{i}^{\prime}$ be such that $f\left(p_{i}\right)$ is the unique element of $L_{0} \cap L_{1}$. Then $L_{1}^{\prime} \cup\left\{x \wedge p_{1}: x \in L_{0}^{\prime} \backslash\left\{p_{0}\right\}\right\}$ is a faithful pre-image of $L$ by $f$.

(iii) Again it suffices to consider $n=2$. Let $T$ be a smooth subsemilattice of $(L ; \wedge)$.

If $T \subseteq L_{i}$ for some $i<2$, let $\rho_{i}$ be a retraction of $L_{i}$ onto $T$. A retraction $\rho$ of $L$ onto $T$ is then given by

$$
\rho(x)= \begin{cases}\rho_{i}(x) & \text { if } x \in L_{i}, \\ 0_{T} & \text { if } i=1 \text { and } x \in L_{0}, \\ 1_{T} & \text { if } i=0 \text { and } x \in L_{1},\end{cases}
$$

where $0_{T}, 1_{T}$ denote respectively the zero and unit of $T$.

If there is no $i<2$ with $T \subseteq L_{i}$, let $L_{0} \cap L_{1}=\{p\}$ and note that $L_{1} \cap T$ and $\left(L_{0} \cap T\right) \cup\{p\}$ are smooth $\wedge$-subsemilattices of $L_{1}$ and $L_{0}$ respectively. Hence there exist retractions $\rho_{1}$ of $L_{1}$ onto $L_{1} \cap T$ and $\rho_{0}$ of $L_{0}$ onto $\left(L_{0} \cap T\right) \cup\{p\}$. A retraction $\rho$ of $L$ onto $T$ is then given by

$$
\rho(x)= \begin{cases}\wedge\left(L_{1} \cap T\right) & \text { if } x \in L_{0} \backslash\{p\} \text { and } \rho_{0}(x)=p, \\ \rho_{0}(x) & \text { if } x \in L_{0} \backslash\{p\} \text { and } \rho_{0}(x) \neq p, \\ \rho_{1}(x) & \text { otherwise. }\end{cases}
$$


A minor modification of the proof of Lemma 4.1(ii) yields the following.

Lemma 4.2. Let $S$ be a bounded semilattice and $\mathbf{K}$ a class of semilattices. If $S \backslash\{1\}$ is faithfully pre-imaged over $\mathbf{K}$, then so is $S$.

Proposition 4.3. $\operatorname{End}(L ; \wedge)$ is regular for all $L \in \mathbf{B}$.

Proof. Let $L=\Sigma_{V}\left(L_{i}: i<n\right)$ where each $L_{i}$ is a finite capped binary tree. Let $f \in \operatorname{End}(L ; \wedge)$.

Inasmuch as every bounded $\wedge$-subsemilattice of $L$ is smooth, Lemma 2.3 and (a superficial use of) Lemma 4.1 (iii) provide a retraction of $(L ; \wedge)$ onto $\operatorname{Im}(f)$.

Because the strong meet property is not affected by adjoining a unit to a semilattice, Lemma 3.3 implies that each $L_{i}$ satisfies the strong meet property, whence, by Lemma $4.1(\mathrm{i}), L$ satisfies it as well. Clearly $\operatorname{Im}(f) \in \mathbf{B}$, hence Lemmas 3.2 and 4.2, in conjunction with Lemma 4.1(ii), now ensure that $\operatorname{Im}(f)$ is faithfully pre-imaged by $f$.

As both conditions of Proposition 2.2 are now established for $f, \operatorname{End}(L ; \wedge)$ is regular.

The proof of the next lemma requires the following observation about semilattices $S$ satisfying the strong meet property: if $R$ is a finite, nonvoid subset of $S$, there exist $a, b \in R$ (not necessarily distinct) such that $\bigwedge R=a \wedge b$. The crucial step in an inductive proof of this statement is that if $R$ contains elements $a, b, c$ such that $\Lambda(R \backslash\{c\})=a \wedge b$ and $c \| a \wedge b$ and $a \ngtr c$, then $\bigwedge R=c \wedge(a \wedge b)=c \wedge a$ by the strong meet property.

Lemma 4.4. For all $L \in \mathbf{B}^{d}$, every smooth $\wedge$-subsemilattice of $L$ is a $\wedge$-retract of $L$.

Proof. By Lemma 4.1(iii) it suffices to assume that $L$ is the dual of a finite capped binary tree. Let $T$ be a smooth $\wedge$-subsemilattice of $L$.

We first establish the implication

$$
\text { for all } a, b, x \in T, x<a \vee_{T} b \text { implies } x<a \vee b \text {. }
$$

Indeed, let $a, b, x \in T$ such that $x<a \vee_{T} b$. Then $x \geq a \vee b$, for $x \geq a \vee b$ would imply (since $x \in T$ ) that $x \geq a \vee_{T} b$. Thus $x \| a \vee b$ or $x<a \vee b$. Were $x \| a \vee b$, then $T$ would not be smooth. Hence $x<a \vee b$ and (*) is established.

Define a mapping $\rho: L \rightarrow L$ by $\rho(x)=\bigvee_{T}((x] \cap T)$ for all $x \in L$. By Lemma 2.3, it is sufficient to assume that not all elements of $T$ are comparable and so $0 \in T$. In particular, $\rho$ is well defined. It will be shown that $\rho$ is a retraction of $L$ onto $T$.

Clearly, $\rho$ is order-preserving, $\operatorname{Im}(\rho)=T$, and $\rho(x)=x$ for $x \in T$. Thus, it remains to show that, for $x, y \in L$, if $x \| y$ then $\rho(x) \wedge \rho(y)=0$. Suppose $x \| y$ and $\rho(x) \wedge \rho(y) \neq 0$. Then $\rho(x)$ is comparable with $\rho(y)$. Say, with no loss in generality, that $0<\rho(x) \leq \rho(y)$. Then $0<z$ for some $z \in(x] \cap T$. Since $z \leq \rho(x)$, it follows that $z \leq \rho(y)$. If $z=\rho(y)$, then $0<\sigma(y) \leq \rho(y)=z$ where $\sigma(y)=\bigvee((y] \cap T)$. In which case, since $\sigma(y) \leq y$ and $z \leq x, \sigma(y) \leq x \wedge y$, which is absurd as $x \wedge y=0$. Thus, it must be that $z<\rho(y)$. However, by Lemma 3.3 and the observation preceding this lemma, $\rho(y)=a \vee_{T} b$ for some (not necessarily distinct) $a, b \in(y] \cap T$. In particular, 
$z<a \vee_{T} b$ and, hence, $z<a \vee b \leq y$ by $(*)$. It follows that $z \leq x \wedge y=0$, which is impossible. Hence $\rho$ is a retraction.

Proposition 4.5. $\operatorname{End}(L ; \wedge)$ is regular for all $L \in \mathbf{B}^{d}$.

Proof. Let $L=\Sigma_{V}\left(L_{i}: i<n\right)$ where each $L_{i}$ is the dual of a finite capped binary tree. For $i<n$ let $u_{i}$ denote the zero of $L_{i}$.

Let $f \in \operatorname{End}(L ; \wedge)$. In view of Proposition 2.2, we first show that $\operatorname{Im}(f)$ is a $\wedge$-retract of $L$. By Lemma 4.4 it suffices to prove that $\operatorname{Im}(f)$ is a smooth $\wedge$ subsemilattice of $L$. Suppose, to the contrary, that there exist $a, b, c \in \operatorname{Im}(f)$ such that $c \| a \vee b$ and $c<a \vee_{T} b$, where $T=\operatorname{Im}(f)$. Clearly, $a \| b$ since otherwise $a \vee b=a \vee_{T} b$. Thus, $a, b \in L_{j}$ for some $j<n$ and, since $a \vee b \| c, c \in L_{j}$ too. Clearly, $a \nsupseteq c$ as $a \vee b \| c$ and, were it the case that $a<c$, then $[a) \cap L_{j}$ would contain two incomparable elements (c and $\left.a \vee b\right)$, which is absurd. Thus, $a \| c$ and, likewise, $b \| c$. In particular, $a, b, c$ form an antichain.

Choose $a^{\prime} \in f^{-1}\{a\}, b^{\prime} \in f^{-1}\{b\}$, and $c^{\prime} \in f^{-1}\{c\}$. Since $a, b, c$ form an antichain, so do $a^{\prime}, b^{\prime}, c^{\prime}$. Thus, for some $k<n, a^{\prime}, b^{\prime}, c^{\prime} \in L_{k}$ and, in particular, $f\left(u_{k}\right)=u_{j}$.

Observe that

$$
f\left(a^{\prime} \vee b^{\prime}\right) \geq f\left(a^{\prime}\right) \vee_{T} f\left(b^{\prime}\right)=a \vee_{T} b>c .
$$

If $c^{\prime} \| a^{\prime} \vee b^{\prime}$, then $c^{\prime} \wedge\left(a^{\prime} \vee b^{\prime}\right)=u_{k}$ and, hence, $c \wedge f\left(a^{\prime} \vee b^{\prime}\right)=u_{j}$, which violates $(*)$. Thus, $c^{\prime}$ is comparable with $a^{\prime} \vee b^{\prime}$. If $c^{\prime} \geq a^{\prime} \vee b^{\prime}$, then by $(*)$, $c>c$, which is absurd. Thus we conclude that $c^{\prime}<a^{\prime} \vee b^{\prime}$.

Since $\left\{a^{\prime} \vee c^{\prime}, b^{\prime} \vee c^{\prime}\right\} \subseteq\left[c^{\prime}\right) \cap L_{k}$, it follows that $a^{\prime} \vee c^{\prime}$ is comparable with $b^{\prime} \vee c^{\prime}$. Suppose $a^{\prime} \vee c^{\prime}=b^{\prime} \vee c^{\prime}$. Since $c^{\prime}<a^{\prime} \vee b^{\prime}$, this would imply

$$
b^{\prime} \vee c^{\prime} \leq a^{\prime} \vee b^{\prime} \leq\left(a^{\prime} \vee c^{\prime}\right) \vee\left(b^{\prime} \vee c^{\prime}\right)=b^{\prime} \vee c^{\prime},
$$

whereupon $a^{\prime} \vee b^{\prime}=a^{\prime} \vee c^{\prime}=b^{\prime} \vee c^{\prime}$. As the antichain $\left\{a^{\prime}, b^{\prime}, c^{\prime}\right\}$ lies in $L_{k}$ (the dual of a capped binary tree), this is impossible. It follows that $a^{\prime} \vee c^{\prime}$ and $b^{\prime} \vee c^{\prime}$ are distinct as well as comparable. Suppose, with no loss in generality, that $b^{\prime} \vee c^{\prime}<a^{\prime} \vee c^{\prime}$.

Observe that $a^{\prime} \| b^{\prime} \vee c^{\prime}$. To see this, suppose not. If $a^{\prime} \geq b^{\prime} \vee c^{\prime}$, then $a^{\prime} \geq b^{\prime}$, which is absurd. If $a^{\prime}<b^{\prime} \vee c^{\prime}$, then $a^{\prime} \vee c^{\prime} \leq b^{\prime} \vee c^{\prime}$, which is impossible by hypothesis.

Now, $a^{\prime} \| b^{\prime} \vee c^{\prime}$ implies $a^{\prime} \wedge\left(b^{\prime} \vee c^{\prime}\right)=u_{k}$. Hence, $a \wedge f\left(b^{\prime} \vee c^{\prime}\right)=u_{j}$. Because $a>u_{j}$ and $f\left(b^{\prime} \vee c^{\prime}\right) \geq b>u_{j}$, it follows that $a \| f\left(b^{\prime} \vee c^{\prime}\right)$ and therefore $f\left(b^{\prime} \vee c^{\prime}\right) \in L_{j}$. Thus, $f\left(b^{\prime} \vee c^{\prime}\right)$ is comparable with $a \vee b$ since both belong to the chain $[b) \cap L_{j}$. But $f\left(b^{\prime} \vee c^{\prime}\right) \nsupseteq a \vee b$ as $a \| f\left(b^{\prime} \vee c^{\prime}\right)$. Thus $f\left(b^{\prime} \vee c^{\prime}\right)<a \vee b$ and, hence, $c<a \vee b$, which violates the original choice of $a, b, c$.

To show that $\operatorname{Im}(f)$ is regular it remains, by Proposition 2.2, to find a $\wedge$ subsemilattice $T^{\prime}$ of $L$ such that $f \backslash T^{\prime}$ is an isomorphism of $T^{\prime}$ onto $\operatorname{Im}(f)$.

Set $U=\left\{u_{i}: i<n\right\}$. For $x \in \operatorname{Im}(f)$, choose $x^{\prime} \in f^{-1}\{x\}$ as follows: let $x^{\prime}=\bigvee\left(U \cap f^{-1}\{x\}\right)$ unless $U \cap f^{-1}\{x\}=\varnothing$, in which case choose $x^{\prime}$ arbitrarily in $f^{-1}\{x\}$. Let $T=\left\{x^{\prime}: x \in \operatorname{Im}(f)\right\}$.

It must be shown that $T$ is closed under meets. However, if $x^{\prime} \| y^{\prime}$, then $x^{\prime} \wedge y^{\prime}=u_{i}$ for some $0 \leq i<n$. In particular, $x \wedge y=f\left(u_{i}\right)$ and $u_{i} \in$ $f^{-1}(x \wedge y)$. It follows that $u_{i}=(x \wedge y)^{\prime}$. 


\section{The ClASS $\mathbf{R}$}

Given bounded lattices $L_{i}, i<n$, where $n>1$, their horizontal sum is defined (only up to isomorphism) as follows. First replace each $L_{i}$ by an isomorphic copy $L_{i}^{\prime}$ such that $L_{i}^{\prime} \cap L_{j}^{\prime}=\varnothing$ whenever $i \neq j$, and choose 0,1 to be any objects not elements of $\bigcup\left(L_{i}^{\prime}: i<n\right)$. A partial order is then defined on $\bigcup\left(L_{i}^{\prime}: i<n\right) \cup\{0,1\}$ by retaining the ordering within each lattice and defining $0<x<1$ for all $x \in \bigcup\left(L_{i}^{\prime}: i<n\right)$. The resulting lattice is denoted $\Sigma_{H}\left(L_{i}: i<n\right)$. In practice, the distinction between $L_{i}$ and $L_{i}^{\prime}$ will be suppressed, that is, the $L_{i}$ will be presumed pairwise disjoint.

A class $\mathbf{K}$ of finite lattices is said to be rectilinearly closed if, for all $n>1$, $\Sigma_{V}\left(L_{i}: i<n\right)$ and $\Sigma_{H}\left(L_{i}: i<n\right)$ both belong to $\mathbf{K}$ whenever $L_{i} \in \mathbf{K}, i<n$.

Let $\mathbf{R}$ denote the intersection of all rectilinearly closed classes that contain the one-element and two-element chains. The goal of this section is to prove that $\operatorname{End}(L ; \wedge)$ is regular for all $L \in \mathbf{R}$. The proof requires a lemma establishing several properties of $\mathbf{R}$, most of which were encountered earlier. An exception is the strong antichain property, defined as follows.

An antichain $A$ in a lattice $L$ is said to be self-disjoint if $a_{0} \wedge a_{1}=b_{0} \wedge b_{1}$ whenever $a_{0}, a_{1}, b_{0}, b_{1}$ are elements of $A$ with $a_{0} \neq a_{1}$ and $b_{0} \neq b_{1}$. We say that $L$ satisfies the strong antichain property if every antichain in $L$ is self-disjoint or contains distinct elements $a, b, c$ such that $a \wedge(b \vee c) \leq b$.

Lemma 5.1. For every $L \in \mathbf{R}$ the following hold.

(i) L satisfies the strong meet property.

(ii) $L$ satisfies the strong antichain property.

(iii) $L$ is faithfully pre-imaged over $\mathbf{R}$.

(iv) Every smooth $\wedge$-subsemilattice of $L$ is a $\wedge$-retract of $L$ and a member of $\mathbf{R}$.

(v) The implications

$$
x\|y \vee z \Rightarrow x\| y \wedge z \Rightarrow x \wedge(y \vee z) \leq y \wedge z
$$

hold for all $x, y, z \in L$ with $y \| z$.

Proof. (i) Because the one- and two-element chains satisfy the strong meet property, it suffices, by Lemma 4.1(i), to assume that $L=\Sigma_{H}\left(L_{i}: i<n\right)$ where each $L_{i}$ satisfies the strong meet property.

Let $a_{j}, b_{j}, j=0,1$, be elements of $L$ such that $a_{0} \| a_{1}$ and $b_{j} \in\left[a_{j}\right) \backslash\left[a_{1-j}\right)$. Clearly none of these elements can be 0 or 1 , hence for $j=0,1$ the summand containing $a_{j}$ also contains $b_{j}$. Hence $a_{0} \wedge a_{1}=b_{0} \wedge b_{1}$ if $a_{0}$ and $a_{1}$ belong to the same summand, while if they belong to different summands, then $a_{0} \wedge a_{1}=$ $0=b_{0} \wedge b_{1}$.

(ii) Because the strong antichain property is trivial for chains and is obviously preserved by vertical sums, it suffices to assume that $L=\Sigma_{H}\left(L_{i}: i<n\right)$ where each $L_{i}$ satisfies the strong antichain property.

If an antichain $A$ in $L$ is neither self-disjoint nor a subset of one of the summands, then there exist $i, j<n$ such that $i \neq j, L_{i} \cap A \neq \varnothing$, and $\left|L_{j} \cap A\right|>1$. Taking $a \in L_{i} \cap A$ and $b, c \in L_{j} \cap A$ with $b \neq c$, we have $a \wedge(b \vee c)=0<b$.

(iii) By Lemma 3.1 and Lemma 4.1(ii), we assume $L=\sum_{H}\left(L_{i}: i<n\right)$ where each $L_{i}$ is faithfully pre-imaged over $\mathbf{R}$. 
Let $T \in \mathbf{R}$ and $f \in \operatorname{End}(T ; \wedge)$ such that $(L ; \wedge)$ is a subsemilattice of $(\operatorname{Im}(f) ; \wedge)$. For $i<n$, let $A_{i}$ be a faithful pre-image of $L_{i}$ by $f$, and let $z_{i}$ be the element of $A_{i}$ such that $f\left(z_{i}\right)=0_{i}$, the zero of $L_{i}$.

If the antichain $\left\{z_{i}: i<n\right\}$ is not self-disjoint, then, by (ii), the strong antichain property yields distinct $j, k, l \in I$ such that $z_{j} \wedge\left(z_{k} \vee z_{l}\right) \leq z_{k}$. It follows that $0_{k}=f\left(z_{k}\right) \geq f\left(z_{j}\right) \wedge f\left(z_{k} \vee z_{l}\right) \geq 0_{j} \wedge\left(0_{k} \vee 0_{l}\right)=0_{j} \wedge 1=0_{j}$, which is absurd. Hence $\left\{z_{i}: i<n\right\}$ is self-disjoint, and therefore we may set $z=z_{i} \wedge z_{j}$ for all $i, j \in I$ with $i \neq j$. Clearly, $f(z)=0$.

By (i), the strong meet property gives $a \wedge b=z$ whenever $a \in A_{i}, b \in A_{j}$ and $i \neq j$. Thus $\bigcup\left(A_{i}: i \in I\right) \cup\{z\}$ is a subsemilattice of $T$ and hence a faithful pre-image of $L \backslash\{1\}$ by $f$. Finally, $L$ is faithfully pre-imaged by Lemma 4.2.

(iv) As the statement is trivial for the one- and two-element chains, there are two cases to consider.

Case 1. Suppose $L=\Sigma_{V}\left(L_{i}: i<n\right)$ where for each $i$, every smooth $\wedge$ subsemilattice of $L_{i}$ is a $\wedge$-retract of $L_{i}$ and a member of $\mathbf{R}$. Let $T$ be a smooth $\wedge$-subsemilattice of $L$. By Lemma 4.1(iii), $T$ is a $\wedge$-retract of $L$. To prove that $T \in \mathbf{R}$, it suffices to take $n=2$ and note that if $T$ is not a subset of $L_{0}$ or $L_{1}$, then $T \cong\left(\left(L_{0} \cap T\right) \cup\{p\}\right)+{ }_{V}\left(L_{1} \cap T\right)$ or $T \cong\left(L_{0} \cap T\right)+{ }_{V} 2+{ }_{V}\left(L_{1} \cap T\right)$, where $L_{0} \cap L_{1}=\{p\}$.

Case 2. Suppose $L=\Sigma_{H}\left(L_{i}: i<n\right)$, with each $L_{i}$ as in Case 1 , and let $T$ be a smooth $\wedge$-subsemilattice of $L$. If $T \subseteq L_{i} \cup\{0,1\}$ for some $i<n$, then by Lemma 4.1(iii) there is a $\wedge$-retraction of $L_{i} \cup\{0,1\}$ onto $T$, which can be extended to a $\wedge$-retraction of $L$ onto $T$ by sending all the other elements of $L$ to $\bigwedge T$; moreover, $T \in \mathbf{R}$ by Case 1 .

Setting $I=\left\{i: i<n\right.$ and $\left.L_{i} \cap T \neq \varnothing\right\}$, we may now assume that $|I|>1$, whence in particular $\{0,1\} \subseteq T$. If there exists $i \in I$ such that $L_{i} \cap T$ is not bounded, then, choosing $a$ and $b$ to be distinct maximal elements of $L_{i}$, and choosing $c \in L_{j}$ for some $j \neq i$, we have $c \| a \vee b$ and $c<1=a \vee_{T} b$, contradicting the fact that $T$ is smooth. Thus, $L_{i} \cap T$ is bounded for all $i \in I$ and is therefore a smooth $\wedge$-subsemilattice of $L_{i}$. Hence for all $i \in I$, $L_{i} \cap T \in \mathbf{R}$ and there is a $\wedge$-retraction $\rho_{i}$ of $L_{i}$ onto $L_{i} \cap T$. A $\wedge$-retraction of $L$ onto $T$ is then given by

$$
\rho(x)= \begin{cases}\rho_{i}(x) & \text { if } x \in L_{i}, i \in I, \\ 1 & \text { if } x=1, \\ 0 & \text { otherwise. }\end{cases}
$$

Moreover, $T=\sum_{H}\left(L_{i} \cap T: i \in I\right)$ and thus $T \in \mathbf{R}$.

(v) As the given implications are trivial for chains and are obviously preserved by vertical sums, it suffices to assume that $L=\Sigma_{H}\left(L_{i}: i<n\right)$ where the implications hold in each $L_{i}$.

Given $x, y, z \in L$ with $x \| y \vee z$ or $x \| y \wedge z$, there exist $i, j<n$ such that $x \in L_{i}$ and $\{y, z\} \subseteq L_{j}$. By hypothesis we may assume $i \neq j$. The conditions $x \| y \vee z$ and $x \| y \wedge z$ are then equivalent because $x$ is not comparable with any member of $L_{j}$. Moreover, $x \wedge(y \vee z)=0<y \wedge z$.

Proposition 5.2. $\operatorname{End}(L ; \wedge)$ is regular for every $L \in \mathbf{R}$.

Proof. Let $f \in \operatorname{End}(L ; \wedge)$ and set $T=\operatorname{Im}(f)$. We first show that $T$ is a 
smooth $\wedge$-subsemilattice of $L$. Supposing the contrary, let $a, b, c \in T$ such that $c \| a \vee b$ and $c<a \vee_{T} b$.

Choose $a^{\prime} \in f^{-1}\{a\}, b^{\prime} \in f^{-1}\{b\}$, and $c^{\prime} \in f^{-1}\{c\}$. Since $c \| a \vee b$, Lemma 5.1(v) implies $c \| a \wedge b$ and therefore $c^{\prime} \| a^{\prime} \wedge b^{\prime}$, whereupon (by Lemma 5.1(v) again) $c^{\prime} \wedge\left(a^{\prime} \vee b^{\prime}\right) \leq a^{\prime} \wedge b^{\prime}$. Hence $a \wedge b \geq f\left(c^{\prime} \wedge\left(a^{\prime} \vee b^{\prime}\right)\right)=c \wedge f\left(a^{\prime} \vee b^{\prime}\right)$. However, $a \vee_{T} b>c$ and thus $f\left(a^{\prime} \vee b^{\prime}\right)>c$, so $a \wedge b \geq c \wedge f\left(a^{\prime} \vee b^{\prime}\right)=c$, contradicting the fact that $c \| a \wedge b$.

Because $T$ is smooth, Lemma 5.1(iv) yields the first condition of Proposition 2.2 and also implies that $T \in \mathbf{R}$. Applying Lemma 5.1(iii) to $T$ establishes the second condition of Proposition 2.2, and thus $f$ is regular.

\section{FinIte LATTICES WHOSE $\wedge$-ENDOMORPHISMS ARE NOT REGULAR}

The objective of this section is to show that, for a finite lattice $L$, if $\operatorname{End}(L ; \wedge)$ is regular, then $L \in \mathbf{B} \cup \mathbf{B}^{d} \cup \mathbf{R}$. The strategy is two-fold. Set $\mathbf{L}=\left\{\mathscr{L}_{i}: i<\right.$ $8\} \cup\left\{\mathscr{L}_{i}, \mathscr{L}_{i}{ }^{d}: 8 \leq i<24\right\}$ where, for $i<24, \mathscr{L}_{i}$ is as shown in Figure 1 on pages 658 and 659 . The first step is to show that, for a finite lattice $L$, if $L$ has a sublattice isomorphic to a member of $\mathbf{L}$, then $\operatorname{End}(L ; \wedge)$ is not regular (Proposition 6.2). The seccnd step is to show that if $L$ does not have a sublattice isomorphic to a member of $\mathbf{L}$, then $L \in \mathbf{B} \cup \mathbf{B}^{d} \cup \mathbf{R}$ (Proposition 6.8).

In view of Propositions 4.3, 4.4, and 5.2, it follows from Propositions 6.2 and 6.8 that, for a finite lattice $L, \operatorname{End}(L ; \wedge)$ is regular if and only if $L \in \mathbf{B} \cup \mathbf{B}^{d} \cup \mathbf{R}$.

Lemma 6.1. Let $L_{0}$ be a finite sublattice of a lattice $L_{1}$. If $\operatorname{End}\left(L_{1} ; \wedge\right)$ is regular, then $\operatorname{End}\left(L_{0} ; \wedge\right)$ is regular.

Proof. Let $f_{0} \in \operatorname{End}\left(L_{0} ; \wedge\right)$ and choose (by Lemma 2.3) a retraction $\rho$ of $\left(L_{1} ; \wedge\right)$ onto $\left(L_{0} ; \wedge\right)$. Set $f_{1}=f_{0} \rho \in \operatorname{End}\left(L_{1} ; \wedge\right)$ and note that $\operatorname{Im}\left(f_{1}\right)=$ $\operatorname{Im}\left(f_{0}\right)$.

By Proposition 2.2, $\operatorname{Im}\left(f_{1}\right)$ is a retract of $\left(L_{1} ; \wedge\right)$, and there exists a subsemilattice $S$ of $\left(L_{1} ; \wedge\right)$ such that $S$ is a faithful pre-image of $\operatorname{Im}\left(f_{1}\right)$ by $f_{1}$. It follows that $\operatorname{Im}\left(f_{0}\right)$ is a retract of $\left(L_{0} ; \wedge\right)$, and $\rho(S)$ is a subsemilattice of $\left(L_{0} ; \wedge\right)$ that is a faithful pre-image of $\operatorname{Im}\left(f_{0}\right)$ by $f_{0}$. Hence $f_{0}$ is regular by Proposition 2.2.

Proposition 6.2. For a lattice $L$, if $L$ has a sublattice isomorphic to a member of $\mathbf{L}$, then $\operatorname{End}(L ; \wedge)$ is not regular.

Proof. By Lemma 6.1, it is sufficient to show that every member of $\mathbf{L}$ has a $\wedge$-endomorphism that is not regular.

For $i<8$, we will define $f \in \operatorname{End}\left(\mathscr{L}_{i} ; \wedge\right)$ and leave it as a (fair) exercise to the reader to show that there is no $g \in \operatorname{End}\left(\mathscr{L}_{i} ; \wedge\right)$ such that $f=f g f$.

For $\mathscr{L}_{0}: f(p)=0, f(q)=p, f(r)=q, f(s)=r$, identity elsewhere. For $\mathscr{L}_{1}: f(s)=q, f(t)=r, f(u)=p, f(1)=1$, otherwise map to 0 . For $\mathscr{L}_{2}$ and $\mathscr{L}_{3}: f(r)=r, f(s)=p, f(t)=q, f(1)=1$, otherwise map to 0 . For $\mathscr{L}_{4}$ and $\mathscr{L}_{5}: f(s)=p, f(t)=q, f(u)=r, f(1)=1$, otherwise map to 0 . For $\mathscr{L}_{6}$ and $\mathscr{L}_{7}: f(q)=u, f(r)=v, f(s)=w, f(p)=f(0)=t$, otherwise map to 1 .

For $8 \leq i<24$, we will define $f \in \operatorname{End}\left(\mathscr{L}_{i} ; \wedge\right)$ and $h \in \operatorname{End}\left(\mathscr{L}_{i} ; \vee\right)$ (that is, $\left.h \in \operatorname{End}\left(\mathscr{L}_{i}^{d} ; \wedge\right)\right)$ and again leave it to the reader to show that there is no $g \in \operatorname{End}\left(\mathscr{L}_{i} ; \wedge\right)$ such that $f=f g f$ and that there is no $g \in \operatorname{End}\left(\mathscr{L}_{i} ; \vee\right)$ such that $h=h g h$. 

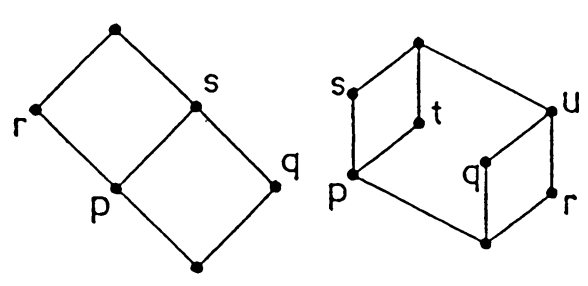

$\mathscr{L}_{0}$

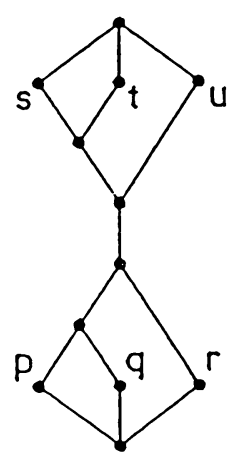

$\mathscr{L}_{4}$

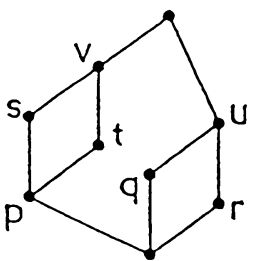

$\mathscr{L}_{8}$

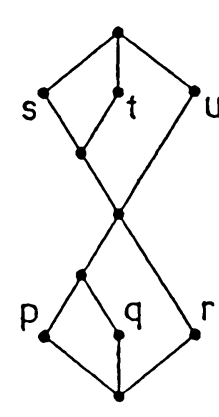

$\mathscr{L}_{5}$

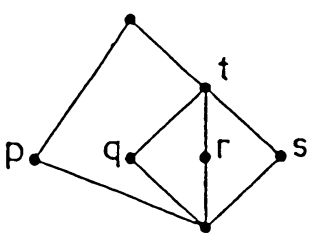

$\mathscr{L}_{9}$

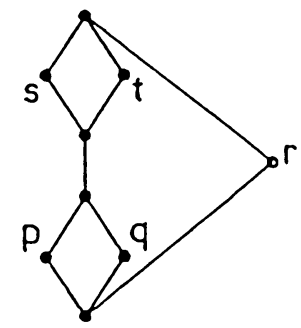

$\mathscr{L}_{2}$

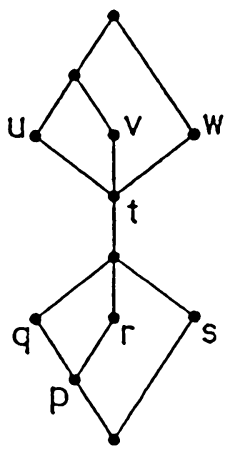

$\mathscr{L}_{6}$

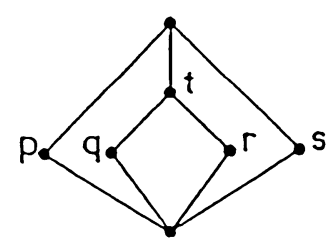

$\mathscr{L}_{10}$

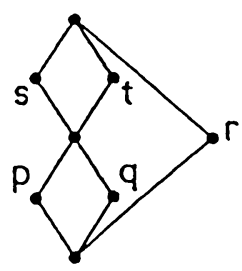

$\mathscr{L}_{3}$

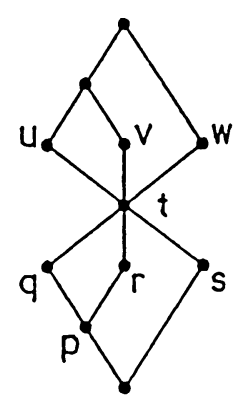

$\mathscr{L}_{7}$

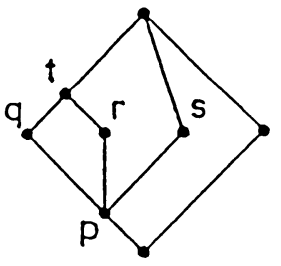

$\mathscr{L}_{11}$
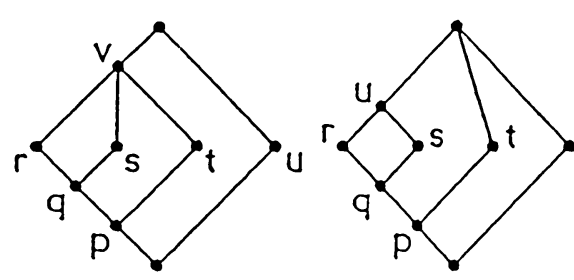

$\mathscr{L}_{13}$

$\mathscr{L}_{12}$
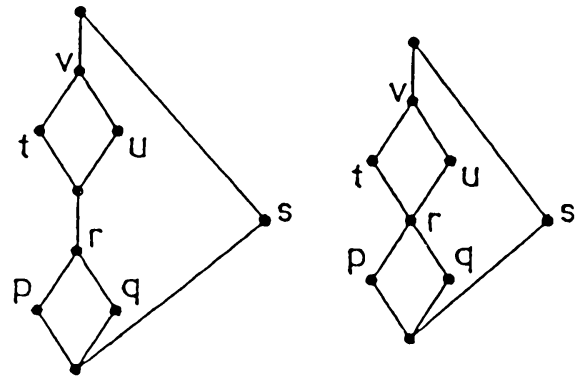


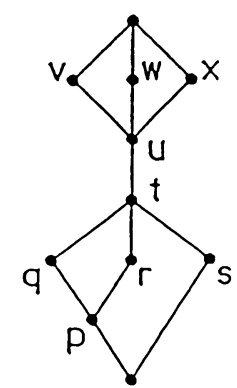

$\mathscr{L}_{16}$

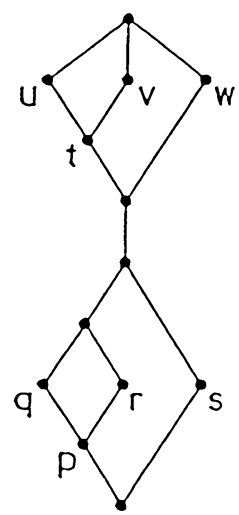

$\mathscr{L}_{20}$

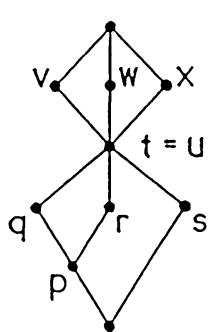

$\mathscr{L}_{17}$

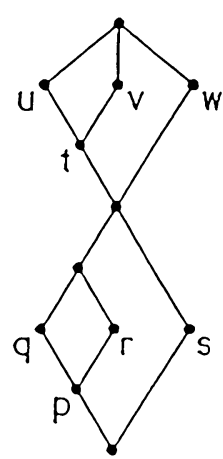

$\mathscr{L}_{21}$

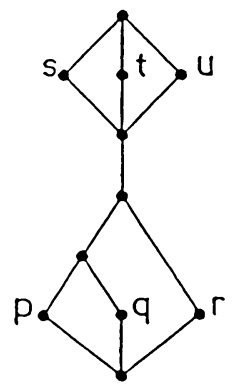

$\mathscr{L}_{18}$

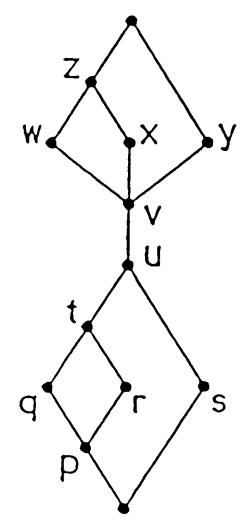

$\mathscr{L}_{22}$

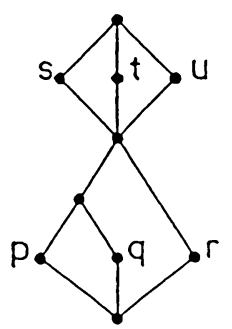

$\mathscr{L}_{19}$

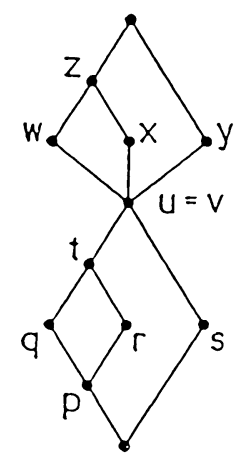

$\mathscr{L}_{23}$

FIGURE 1 (continued)

For $\mathscr{L}_{8}: f(s)=q, f(t)=r, f(u)=p, f(v)=u, f(1)=1$, otherwise map to $0 ; h(p)=u, h(q)=t, h(r)=s, h(u)=v, h(0)=0$, otherwise map to 1 . For $\mathscr{L}_{9}: f(p)=f(0)=0, f(q)=p, f(r)=q, f(s)=r$, and $f(t)=$ $f(1)=1 ; h(p)=q, h(q)=h(1)=t$, identity elsewhere. For $\mathscr{L}_{10}: f(p)=q$, $f(s)=s, f(t)=r, f(1)=1$, otherwise map to $0 ; h(q)=p, h(r)=t$, $h(s)=s, h(0)=0$, otherwise map to 1 . For $\mathscr{L}_{11}: f(q)=p, f(r)=f(t)=q$, $f(s)=r$, identity elsewhere; $h(q)=t, h(r)=s, h(s)=h(t)=1$, identity elsewhere. For $\mathscr{L}_{12}: f(p)=f(u)=0, f(t)=u, f(v)=1$, identity elsewhere; $h(p)=q, h(q)=h(r)=r, h(t)=s, h(u)=t, h(0)=p$, otherwise map to $v$. For $\mathscr{L}_{13}: f(p)=f(r)=f(s)=q, f(t)=s, f(u)=r$, identity elsewhere; $h(q)=p, h(r)=u, h(s)=t, h(t)=h(u)=1$, identity elsewhere. For $\mathscr{L}_{14}: f(s)=s, f(t)=p, f(u)=q, f(v)=r, f(1)=1$, otherwise map to 0 ; $h(p)=t, h(q)=u, h(r)=v, h(s)=s, h(0)=0$, otherwise map to 1. For $\mathscr{L}_{15}$ : see $\mathscr{L}_{14}$. For $\mathscr{L}_{16}: f(p)=f(0)=u, f(q)=v, f(r)=w, f(s)=x$, otherwise map to $1 ; h(v)=q, h(w)=r, h(x)=s, h(1)=t$, otherwise map to 0 . For $\mathscr{L}_{17} ;$ see $\mathscr{L}_{16}$. For $\mathscr{L}_{18}: f(s)=p, f(t)=q, f(u)=r, f(1)=1$, otherwise map to $0 ; h(p)=s, h(q)=t, h(r)=u, h(0)=0$, otherwise map to 1 . For $\mathscr{L}_{19}$ : see $\mathscr{L}_{18}$. For $\mathscr{L}_{20}: f(t)=p, f(u)=q, f(v)=r, f(w)=s$, $f(1)=1$, otherwise map to $0 ; h(p)=t, h(q)=u, h(r)=v, h(s)=w$, $h(0)=0$, otherwise map to 1 . For $\mathscr{L}_{21}$ : see $\mathscr{L}_{20}$. For $\mathscr{L}_{22}: f(p)=f(0)=v$, $f(q)=w, f(r)=x, f(s)=y, f(t)=z$, otherwise map to $1 ; h(w)=q$, 


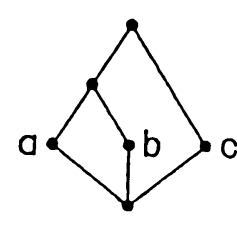

$\mathscr{L}_{24}$

FIGURE 2

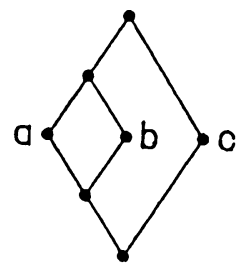

$\mathscr{L}_{25}$

$h(x)=r, h(y)=s, h(z)=t, h(1)=u$, otherwise map to 0 . For $\mathscr{L}_{23}:$ see $\mathscr{L}_{22}$.

Henceforth, let $L$ be a finite lattice such that no sublattice of $L$ is isomorphic to a lattice in $\mathbf{L}$.

For a subset $X \subseteq L$, let $[X]$ denote the sublattice generated by $X$. Further, let $N_{5}$ denote the 5-element nonmodular lattice $\{0, p, q, r, 1\}$ in which $0<$ $p<q<1,0<r<1$, and there is no further ordering.

Lemma 6.3. For distinct $a, b, c \in L$, if $a \| b, b$ is comparable with $c$, and $a \wedge b<c<a \vee b$, then $[\{a, b, c\}] \cong N_{5}$.

Proof. Say, with no loss in generality, that $b<c<a \vee b$. Clearly, $a \vee c=a \vee b$. Suppose that $a \wedge c \neq a \wedge b$. Then $a \wedge b<a \wedge c<a$. Set $d=(a \wedge c) \vee b$. Since $a \wedge c \| b, b<d<a \vee b$. However, $a \wedge d \leq a \wedge c$ and $a \wedge d \geq a \wedge(a \wedge c)=a \wedge c$; that is $a \wedge d=a \wedge c$. It follows that $[\{a, b, a \wedge c\}]$ is isomorphic to $\mathscr{L}_{0}$, which is absurd. Thus $a \wedge c=a \wedge b$ as required.

Lemma 6.4. Let $a, b, c \in L$.

(i) If $a \succ b$ and $c \| a, b$, then $[\{a, b, c\}] \cong N_{5}$.

(ii) If $a \vee b \succ a, b$ and $c \| a \vee b$, then $c \| a, b$. Dually, if $a, b \succ a \wedge b$ and $c \| a \wedge b$, then $c \| a, b$.

Proof. (i) Suppose $a \vee c \neq b \vee c$. Then $a \| b \vee c$ and so $a \wedge(b \vee c)=b$. Since $c<b \vee c<a \vee c$, it follows from Lemma 6.3 that $[\{a, c, b \vee c\}] \cong N_{5}$ and hence $a \wedge c=a \wedge(b \vee c)=b$, which is absurd as $c \| b$. Thus $a \vee c=b \vee c$ and, by a dual argument, $a \wedge c=b \wedge c$ as required.

(ii) Suppose $a \vee b \succ a, b$ and $c \| a \vee b$. Assume, with no loss in generality, that $a$ is comparable with $c$. Since $c \| a \vee b, a, b \nsupseteq c$. Thus $c \geq a$ and, hence, $c \nsupseteq b$. By (i), $c \wedge b=c \wedge(a \vee b) \geq a$, which contradicts $a \| b$.

Lemma 6.5. Let $\mathscr{L}_{24}$ and $\mathscr{L}_{25}$ be given as in Figure 2 and let $a, b, c \in L$. If $a \| b$ and $c \| a \vee b$, then $[\{a, b, c\}]$ is isomorphic to $\mathscr{L}_{24}$ or $\mathscr{L}_{25}$. Dually, if $a \| b$ and $c \| a \wedge b$, then $[\{a, b, c\}]$ is isomorphic to $\mathscr{L}_{24}^{d}$ or $\mathscr{L}_{25}$.

Proof. Suppose $a \| b$ and $c \| a \vee b$. Choose $a_{0}, \ldots, a_{m-1}, b_{0}, \ldots, b_{n-1} \in L$ such that $a \vee b \succ a_{0} \succ a_{1} \succ \cdots \succ a_{m-1} \succ a$ and $a \vee b \succ b_{0} \succ b_{1} \succ \cdots \succ b_{n-1} \succ$ $b$. Since $c \| a \vee b, a, b \nsupseteq c$ and either $c \nsupseteq a$ or $c \nsupseteq b$. Suppose, with no loss in generality, that $c \nsupseteq a$. Then, in particular, $c \| a \vee b, a_{0}, \ldots, a_{m-1}, a$. By Lemma 6.4(i),

$$
c \wedge(a \vee b)=c \wedge a_{0}=c \wedge a_{1}=\cdots=c \wedge a_{m-1}=c \wedge a
$$

and

$$
c \vee(a \vee b)=c \vee a_{0}=c \vee a_{1}=\cdots=c \vee a_{m-1}=c \vee a
$$


By Lemma 6.4(ii), $c \| b_{0}$ and, hence, $c \wedge(a \vee b)=c \wedge b_{0}$ by Lemma 6.4(i). Were it the case that $c \geq b$, then it would follow that $b \leq c \wedge(a \vee b)=c \wedge a$ which violates $a \| b$. Thus $c \| b$. Hence, $c \| a \vee b, b_{0}, \ldots, b_{n-1}, b$ and, by Lemma 6.4(i),

$$
c \wedge(a \vee b)=c \wedge b_{0}=c \wedge b_{1}=\cdots=c \wedge b_{m-1}=c \wedge b
$$

and

$$
c \vee(a \vee b)=c \vee b_{0}=c \vee b_{1}=\cdots=c \vee b_{m-1}=c \vee b .
$$

Thus, $a \vee b<c \vee(a \vee b)=c \vee a=c \vee b$ and $c \wedge(a \vee b)=c \wedge a=c \wedge b=$ $(c \wedge a) \wedge(c \wedge b)=c \wedge(a \wedge b)$. There are two possibilities depending on whether $c$ is comparable to $a \wedge b$ or not. If $c$ is comparable to $a \wedge b$, then $[\{a, b, c\}] \cong \mathscr{L}_{24}$. Otherwise, $a \vee b>a \wedge b>c \wedge(a \wedge b)=c \wedge(a \vee b)$. Thus $[\{c, a \vee b, a \wedge b\}] \cong N_{5}$ by Lemma 6.3 and, in particular, $c \vee(a \wedge b)=c \vee(a \vee b)$. It follows that $[\{a, b, c\}] \cong \mathscr{L}_{25}$.

$L$ is vertically decomposable providing $L=\Sigma_{V}\left(L_{i}: i<n\right)$ where $\left|L_{i}\right|>1$ for all $i$, and $L$ is horizontally decomposable if $L=\Sigma_{H}\left(L_{i}: i<n\right)$. In the event that $L$ is either vertically or horizontally decomposable then it is said to be rectilinearly decomposable.

For $a \leq b$ in $L$, let $[a, b]$ denote $\{x: a \leq x \leq b\}$.

Lemma 6.6. If there exist $a, b \in L$ with $a \| b$ and $0<a \wedge b<a \vee b<1$, then $L$ is rectilinearly decomposable.

Proof. Suppose $0<a \wedge b<a \vee b<1$ for some $a, b \in L$ with $a \| b$, and that $L$ is not vertically decomposable. It is to be shown that $L$ is horizontally decomposable.

Since $L$ is finite, $a$ and $b$ may be chosen such that whenever $a \wedge b, a \vee b \in$ $[c \wedge d, c \vee d]$ with $c \| d$ and $0<c \wedge d<c \vee d<1$, then $a \wedge b=c \wedge d$ and $a \vee b=c \vee d$. Set

$$
L_{0}=((a \wedge b] \cup[a \wedge b, a \vee b] \cup[a \vee b)) \backslash\{0,1\}
$$

Since $L$ is not vertically decomposable, $c \in L \backslash L_{0}$ for some $c \neq 0,1$. There are two possibilities: (i) $c \| a \vee b$; (ii) $c \| a \wedge b$.

(i) $c \| a \vee b$. Since $a \| b,[\{a, b, c\}] \cong \mathscr{L}_{24}$ or $\mathscr{L}_{25}$ by Lemma 6.5.

If $[\{a, b, c\}] \cong \mathscr{L}_{24}$, then $(a \vee b) \vee c=1$ by the choice of $a$ and $b$. Since $L$ is not vertically decomposable, there exists $d \| a \wedge b$. By Lemma 6.5, it follows from $a \wedge b=(a \vee b) \wedge c$ that $[\{a \vee b, c, d\}] \cong \mathscr{L}_{24}^{d}$ or $\mathscr{L}_{25}$. Since $(a \vee b) \vee c=1$, it must be isomorphic to $\mathscr{L}_{24}^{d}$. Consequently $[\{a, b, c, d\}] \cong \mathscr{L}_{11}$, which is absurd. Thus, $[\{a, b, c\}] \not \mathscr{L}_{24}$, and so $[\{a, b, c\}] \cong \mathscr{L}_{25}$.

Supposing $(a \wedge b) \wedge c \neq 0$, then $(a \wedge b) \vee c=(a \vee b) \vee c=1$ by the choice of $a$ and $b$. Further, by hypothesis, there exists $e \|(a \wedge b) \wedge c=(a \vee b) \wedge c$. By Lemma 6.5, $[\{a \vee b, c, e\}] \cong \mathscr{L}_{24}^{d}$ or $\mathscr{L}_{25}$. Since $(a \vee b) \vee c=1$, it must be isomorphic to $\mathscr{L}_{24}^{d}$. Therefore $[\{a, b, c, e\}] \cong \mathscr{L}_{13}$, which is impossible. Thus, $(a \wedge b) \wedge c=0$. Furthermore, an argument dual to that just given shows $(a \vee b) \vee c=1$. Hence, to summarize, not only is $[\{a, b, c\}] \cong \mathscr{L}_{25}$ but $(a \wedge b) \wedge c=0$ and $(a \vee b) \vee c=1$.

(ii) $c \| a \wedge b$. The conclusion of (i) is self-dual. Thus, a dual argument leads to the same conclusion.

It now follows that $c \| x$ for any $x \in L_{0}$. Further, since $L_{0}$ is finite, repeated applications of Lemma 6.4(i) show $c \vee x=1$ and $c \wedge x=0$ for every $x \in L_{0}$. 
We claim that both $L \backslash L_{0}$ and $L_{0}$ are sublattices of $L$. Note that $c$ was chosen to be any element of $L \backslash\left(L_{0} \cup\{0,1\}\right)$. Thus, it follows from the preceding paragraph that $d \wedge e, d \vee e \notin L_{0}$ for any $d, e \in L \backslash L_{0}$. In particular, $L \backslash L_{0}$ is a sublattice of $L$. To see that $L_{0}$ is a sublattice of $L$, it is enough to show that $L_{0}$ has both a minimum and a maximum element. Were $L_{0}$ not to have a minimum element, there would exist distinct minimal elements $d, e$ such that $[\{a, b, c, d, e\}] \cong \mathscr{L}_{14}$ or $\mathscr{L}_{15}$, which is absurd. Likewise, a dual argument shows that $L_{0}$ does not have distinct maximal elements.

Consider now the sublattice $L \backslash L_{0}$ of $L$. If $L \backslash L_{0}$ satisfies the hypothesis of the lemma, then repeat the above process to obtain a sublattice $L_{1}$. Next consider the sublattice $L \backslash\left(L_{0} \cup L_{1}\right)$. Continuing in this fashion as far as possible, we obtain sublattices $L_{0}, \ldots, L_{m-1}$ for some $m>0$.

Set $L^{\prime}=L \backslash \bigcup\left(L_{i}: i<m\right)$. If $L^{\prime}=\{0,1\}$, then $m>1$ (since $L \backslash L_{0} \neq$ $\{0,1\})$ and $L=\Sigma_{H}\left(L_{i}: i<m\right)$ as required.

Alternately, suppose $L^{\prime} \backslash\{0,1\} \neq \varnothing$. For $c, d \in L^{\prime} \backslash\{0,1\}$, if $c \| d$, then, by hypothesis, either $c \vee d=1$ or $c \wedge d=0$. Suppose that for some such pair $c \vee d \neq 1$. Then $[\{a, b, c, d\}] \cong \mathscr{L}_{8}$ which is absurd. Likewise, a dual argument shows that $c \wedge d \neq 0$ is also impossible. Thus, for $c, d \in L^{\prime} \backslash\{0,1\}$, if $c \| d$, then $c \vee d=1$ and $c \wedge d=0$. In particular, $L^{\prime}=\Sigma_{H}\left(L_{i}: m \leq i<n\right)$ where each $L_{i}$ is a chain. It follows that, for some $n>1, L=\Sigma_{H}\left(L_{i}: i<n\right)$ as required.

Lemma 6.7. If $L$ is not rectilinearly decomposable, then $L$ is either a capped binary tree or the dual of a capped binary tree.

Proof. We first show that $L$ is either a capped tree (that is, the lattice obtained by adjoining a unit to a tree) or the dual of a capped tree.

Suppose $L$ is neither a capped tree nor the dual of a capped tree. Then there exists a $\vee$-reducible element other than 1 and a $\wedge$-reducible element other than 0 . Choose $a, b, c, d \in L$ such that $a\|b, c\| d, a \vee b$ is a maximal $\vee$-reducible element $<1$, and $c \wedge d$ is a minimal $\wedge$-reducible element $>0$. By Lemma 6.6, for any $x, y \in L$, if $x \| y$, then either $x \wedge y=0$ or $x \vee y=1$. In particular, $a \wedge b=0$ and $c \vee d=1$.

Suppose $c \| a \vee b$. By Lemma 6.5 and the fact that $a \wedge b=0,[\{a, b, c\}] \cong$ $\mathscr{L}_{24}$; moreover, $c \vee(a \vee b)=1$ by the choice of $a$ and $b$. Since $c \| a \vee b$, $c \wedge d \ngtr a \vee b$ and, as $(a \vee b) \wedge c=0, a \vee b \nsupseteq c \wedge d$. Thus, $c \wedge d \| a \vee b$ and, by Lemma $6.5,[\{a, b, c \wedge d\}] \cong \mathscr{L}_{24}$ with $(c \wedge d) \vee(a \vee b)=1$ by the choice of $a$ and $b$. Since $(a \vee b) \vee(c \wedge d)=1, d \nsucceq a \vee b$ and, since $a \vee b \nsupseteq c \wedge d, a \vee b \nsupseteq d$. Thus, in turn, $d \| a \vee b$ and, again by Lemma $6.5,[\{a, b, d\}] \cong \mathscr{L}_{24}$ with $d \vee(a \vee b)=1$ by the choice of $a$ and $b$. To summarize, $[\{a, b, c, d\}] \cong \mathscr{L}_{1}$, which is absurd. Thus, contrary to assumption, $c$ is comparable with $a \vee b$. A similar argument now shows that $d$ is also comparable to $a \vee b$.

Because $c \| d$ and $c, d$ are comparable to $a \vee b$, it follows that $c, d \leq a \vee b$ or $c, d \geq a \vee b$. But since $c \vee d=1 \neq a \vee b$, it cannot be that $c, d \leq a \vee b$. Thus $c, d \geq a \vee b$, that is, $a \vee b \leq c \wedge d$. As $L$ is not vertically decomposable, there exists $e \in L$ such that $e \| a \vee b$. By the choice of $a$ and $b, e \| c \wedge d$ too. Thus, $[\{a, b, e\}] \cong \mathscr{L}_{24}$ where $e \vee(a \vee b)=1$, and $[\{c, d, e\}] \cong \mathscr{L}_{24}^{d}$ where $e \wedge(c \wedge d)=0$. Consequently, $[\{a, b, c, d, e\}] \cong \mathscr{L}_{2}$ or $\mathscr{L}_{3}$, which is absurd.

Thus, $L$ is either a capped tree or the dual of a capped tree.

Suppose $L$ is a capped tree. If $L$ is not a binary tree, then it is possible 
to choose $a \in L$ with at least three covers. Since $L$ is not rectilinearly decomposable, it has more than one $\wedge$-reducible element. Let $b$ be some other $\wedge$-reducible element. If $a \| b$, then $a \wedge b$ is a $\wedge$-reducible element which is distinct from $a$. Thus, with no loss in generality, $b$ may be chosen to be comparable with $a$. It follows that $L$ contains a sublattice isomorphic to $\mathscr{L}_{9}^{d}$ or $\mathscr{L}_{10}^{d}$, which is absurd.

A dual argument shows that if $L$ is the dual of a capped tree, then it is the dual of a capped binary tree.

Proposition 6.8. Let $L$ be a finite lattice. If $L$ does not contain a sublattice isomorphic to a member of $\mathbf{L}$, then $L \in \mathbf{B} \cup \mathbf{B}^{d} \cup \mathbf{R}$.

Proof. The proof is by induction on $|L|$. Suppose that the proposition holds for all lattices of cardinality smaller than $|L|$.

If $L$ is not rectilinearly decomposable, then $L \in \mathbf{B} \cup \mathbf{B}^{d}$ by Lemma 6.7.

If $L$ is vertically decomposable, then $L=\Sigma_{V}\left(L_{i}: i<n\right)$ where, for every $i<n, L_{i}$ is not vertically decomposable. By the inductive hypothesis, $L_{i} \in$ $\mathbf{B} \cup \mathbf{B}^{d} \cup \mathbf{R}$ for every $i<n$. Further, since $\mathbf{B}, \mathbf{B}^{d}$, and $\mathbf{R}$ are closed under vertical sums, it is enough to show that, for one of $\mathbf{B}, \mathbf{B}^{d}$, or $\mathbf{R}, L_{i}$ is a member for every $i<n$. Suppose that this is not the case and that there exist distinct $i, j<n$ for which $L_{i}$ and $L_{j}$ do not both belong to one of $\mathbf{B}, \mathbf{B}^{d}$, or $\mathbf{R}$. There are three possibilities: (i) $L_{i} \in \mathbf{B} \backslash \mathbf{B}^{d}$ and $L_{j} \in \mathbf{B}^{d} \backslash \mathbf{B}$; (ii) $L_{i} \in \mathbf{B} \backslash \mathbf{R}$ and $L_{j} \in \mathbf{R} \backslash \mathbf{B}$; (iii) $L_{i} \in \mathbf{B}^{d} \backslash \mathbf{R}$ and $L_{j} \in \mathbf{R} \backslash \mathbf{B}^{d}$.

(i) $L_{i} \in \mathbf{B} \backslash \mathbf{B}^{d}$ and $L_{j} \in \mathbf{B}^{d} \backslash \mathbf{B}$. By the choice of $L_{i}$ and $L_{j}, \mathscr{L}_{24}^{d}$ is isomorphic to a sublattice of $L_{i}$ and $\mathscr{L}_{24}$ is isomorphic to a sublattice of $L_{j}$. It follows that $L$ contains a sublattice isomorphic to $\mathscr{L}_{4}, \mathscr{L}_{5}, \mathscr{L}_{6}$, or $\mathscr{L}_{7}$, which is absurd.

(ii) $L_{i} \in \mathbf{B} \backslash \mathbf{R}$ and $L_{j} \in \mathbf{R} \backslash \mathbf{B}$. By the choice of $L_{i}, \mathscr{L}_{24}^{d}$ is isomorphic to a sublattice of $L_{i}$. By the choice of $L_{j}, L_{j}=\Sigma_{H}\left(L_{i}^{\prime}: i<m\right)$ for some $m>1$. If $m>2$, then the five-element nondistributive modular lattice (consisting of 0,1 , and a three-element antichain) is isomorphic to a sublattice of $L_{j}$. Were this the case, then $L$ would contain a sublattice isomorphic to $\mathscr{L}_{16}, \mathscr{L}_{17}, \mathscr{L}_{18}^{d}$, or $\mathscr{L}_{19}^{d}$, which is absurd. The only other possibility is that $m=2$ and $\mathscr{L}_{25}$ is isomorphic to a sublattice of $L_{j}$. But then $L$ contains a sublattice isomorphic to $\mathscr{L}_{20}, \mathscr{L}_{21}, \mathscr{L}_{22}^{d}$, or $\mathscr{L}_{23}^{d}$, which is absurd.

(iii) $L_{i} \in \mathbf{B}^{d} \backslash \mathbf{R}$ and $L_{j} \in \mathbf{R} \backslash \mathbf{B}^{d}$. As in (ii), this is impossible.

Thus, if $L$ is vertically decomposable, then $L \in \mathbf{B} \cup \mathbf{B}^{d} \cup \mathbf{R}$.

The only remaining possibility is that $L$ is horizontally decomposable. Hence, $L=\Sigma_{H}\left(L_{i}: i<n\right)$. By the inductive hypothesis, $L_{i} \in \mathbf{B} \cup \mathbf{B}^{d} \cup \mathbf{R}$ for every $i<n$. If $L_{i} \notin \mathbf{R}$ for some $i<n$, then $L$ contains a sublattice isomorphic to $\mathscr{L}_{12}$ or $\mathscr{L}_{12}^{d}$, which is absurd. Thus, for every $i<n, L_{i} \in \mathbf{R}$ and, in particular, $L \in \mathbf{R}$.

\section{THE MAIN RESUlTS}

Propositions $2.1,3.6,4.3,4.5,5.2,6.2$, and 6.8 yield the following.

Theorem 7.1. For a finite semilattice $S, \operatorname{End}(S ; \wedge)$ is regular if and only if one of the following holds:

(i) $S$ is a binary tree, 


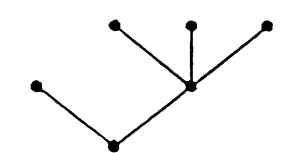

$\mathscr{T}_{0}$

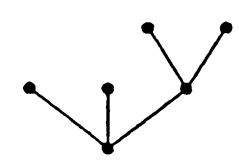

$\mathscr{T}_{1}$

FIGURE 3

(ii) $S$ is a tree with only one $\wedge$-reducible element, or

(iii) $S$ is bounded and, as a lattice, $S \in \mathbf{B} \cup \mathbf{B}^{d} \cup \mathbf{R}$.

Since $\mathbf{B} \cup \mathbf{B}^{d} \cup \mathbf{R}$ is self-dual, the following is an immediate consequence of Theorem 7.1.

Corollary 7.2. For a finite lattice $S, \operatorname{End}(S ; \wedge)$ is regular if and only if $\operatorname{End}(S ; \vee)$ is regular.

The proof of Theorem 7.1(iii) consisted of showing that, for a finite lattice $S, \operatorname{End}(S ; \wedge)$ is regular if and only if $S$ does not contain any member of $\mathbf{L}$ as a sublattice. Set $\mathbf{T}=\left\{\mathscr{T}_{0}, \mathscr{T}_{1}\right\}$ where, for $i<2, \mathscr{T}_{i}$ is given as in Figure 3 . For a tree $S$, it is readily seen that $S$ is binary or has only one $\wedge$-reducible element if and only if $S$ does not contain a member of $\mathbf{T}$ as a subsemilattice. Thus, Theorem 7.1 may be reformulated as follows.

Theorem 7.3. For a finite semilattice $S, \operatorname{End}(S ; \wedge)$ is regular if and only if either

(i) $S$ is a tree that does not contain any member of $\mathbf{T}$ as a subsemilattice, or

(ii) $S$ is a lattice which does not contain any member of $\mathbf{L}$ as a sublattice.

Since no member of $\mathbf{T}$ is a subsemilattice of any other member of $\mathbf{T}$ and no member of $\mathbf{L}$ is a sublattice of any other member of $\mathbf{L}$, it follows that both $T$ (which has two members) and $L$ (which has 40 members) are minimal.

Inspection of Figure 1 reveals the following corollary of Theorem 7.3.

Corollary 7.4. For a finite modular lattice $S, \operatorname{End}(S ; \wedge)$ is regular if and only if $S$ has no sublattice isomorphic to $\mathscr{L}_{0}$.

\section{CONCLUDING REMARK}

Clearly, we have been unable to characterize those infinite semilattices whose endomorphism monoids are regular. Clearly too, the proofs as given are heavily dependent on finiteness. We remark that this is not superficial: for an infinite semilattice $S, \operatorname{End}(S ; \wedge)$ may be regular even though $S$ need be neither a tree nor a lattice. An example is obtained by adjoining three elements $a_{0}, a_{1}, a_{2}$, to the chain $\omega^{*}$ of negative integers and stipulating that $a_{1} \wedge a_{2}=a_{0}$ and $a_{i}<x$ for all $x \in \omega^{*}$ and $i<3$.

\section{ACKNOWLEDGMENTS}

It is a pleasure to thank S. Bulman-Fleming, D. M. Clark, M. Gehrke, S. T. Tschantz, C. Tsinakis, and the referee for their comments and suggestions. 


\section{REFERENCES}

1. M. E. Adams and M. Gould, Posets whose monoids of order-preserving maps are regular, Order 6 (1990), 195-201 (Corrigendum, Order 7 (1990), 105).

2. A. Ya. Aizenshtat, Regular semigroups of endomorphisms of ordered sets, Leningrad. Gos. Ped. Inst. Uchen. Zap. 387 (1968), 3-11; English transl., Amer. Math. Soc. Transl. (2) 139 (1988), 29-35.

3. J. Chvalina, On connected unars with regular endomorphism monoids, Arch. Math. (Brno) 16 (1980), 7-13.

4. L. M. Gluskin, Semigroups of isotone transformations, Uspekhi Mat. Nauk 16 (1961), 157162. (Russian)

5. M. F. Janowitz, Regularity of residuated mappings, Semigroup Forum 42 (1991), 313-332.

6. Proc. Sympos. on Regular Semigroups, Northern Illinois Univ., DeKalb, 1979.

7. B. M. Schein, Ordered sets, semilattices, distributive lattices and Boolean algebras with homomorphic endomorphism semigroups, Fund. Math. 68 (1970), 31-50.

8. L. A. Skornjakov, Unary algebras with regular endomorphism monoids, Acta Sci. Math. 40 (1978), 99-103.

Department of Mathematics, State University of New York, New Paltz, New York 12561

E-mail address: adamsm@snynewvm.bitnet

Department of Mathematics, Vanderbilt University, Nashville, Tennessee 37235

E-mail address: mgould@athena.cas.vanderbilt.edu 